\title{
Tunneling Control of Transmission Coefficient and Group Index in a Quantum Dot Nanostructure
}

\author{
Hamid Reza Hamedi, ${ }^{1}$ A. Khaledi-Nasab, ${ }^{2}$ and H. Ghaforyan ${ }^{3}$ \\ ${ }^{1}$ Institute of Theoretical Physics and Astronomy, Vilnius University, A. Gostauto 12, 01108 Vilnius, Lithuania \\ ${ }^{2}$ Research Institute for Applied Physics, University of Tabriz, Tabriz, Iran \\ ${ }^{3}$ Department of Physics, Payame Noor University, Tehran 193954697, Iran
}

Correspondence should be addressed to Hamid Reza Hamedi; hamid.r.hamedi@gmail.com

Received 15 May 2013; Revised 25 October 2013; Accepted 3 November 2013; Published 29 January 2014

Academic Editor: Oleg Derzhko

Copyright (C) 2014 Hamid Reza Hamedi et al. This is an open access article distributed under the Creative Commons Attribution License, which permits unrestricted use, distribution, and reproduction in any medium, provided the original work is properly cited.

We theoretically study the transmission and group index properties of the probe field in a four-level quantum dot molecule. It is found that the tunnel coupling plays a very important role in realizing the transmission coefficient of the probe field. Moreover, the impact of an incoherent pump field on imaginary part of susceptibility is investigated. We show that probe transmission exhibits oppositional behavior against weak and strong incoherent pump rates. This approach allows substantial flexibility in the manipulation of group velocity of light.

\section{Introduction}

Nowadays, researchers in the field of quantum and nonlinear optics are aware of the importance of quantum coherence and interference [1-10], which can modify the response of the atomic medium to the applied fields. Recently, many interesting physical phenomena based on quantum interference and coherence such as coherent population trapping $[1,2]$, electromagnetically induced transparency (EIT) [36], lasing without inversion (LWI) [7-9], superluminal and subluminal light propagation [11], enhancing Kerr nonlinearity [12], multiwave mixing [13, 14], optical bistability, and multistability [15-17] have been discovered. There are many ways to generate quantum coherence and interference. Generally, they can be realized by coherent driving fields or by initial coherence injections; the interference between different spontaneous emissions paths also can lead to the generation of coherent superposition state. Although the main features of quantum interference processes are adequately covered by the three-level approximation, there has been considerable interest in coherent effects in multilevel systems because the presence of additional levels increases multifold the possibilities of interference phenomena to exist. As we know, light will be considerably absorbed when it passes through an optical medium, which is very inappropriate for the conversion efficiency of nonlinear optical processes. However, the situation changed after Harris and his coworkers discovered the novel phenomenon of EIT in a three-level atomic system in 1990s [4]. They first observed absorption cancellation via EIT. Recently, absorption and dispersion properties of a weak probe field have attracted considerable attention, because they can lead to subluminal and superluminal light propagation. The most important key to successful experiments on controlling the group velocity of light lies in its ability to control optical properties of a medium with coherent and incoherent fields [18]. In one of our recent studies, the absorption-dispersion properties of a four-level Lambda-type atomic system were investigated [19]. It is found that the slope of dispersion not only changes by the intensity of coupling field, but also changes by the rate of an incoherent pump field. Besides the proposals which we mentioned above, there is also significant interest in quantum coherence and interference using quantum dots (QDs) [20-22], because of reports of long dephasing times and preservation of atomic-like properties (e.g., discrete energy levels) at high temperatures, ease of integration, and large bandwidth due to fast carrier. Devices which take advantage of intersubband transitions in quantum dots (QDs) have inherent advantages, 


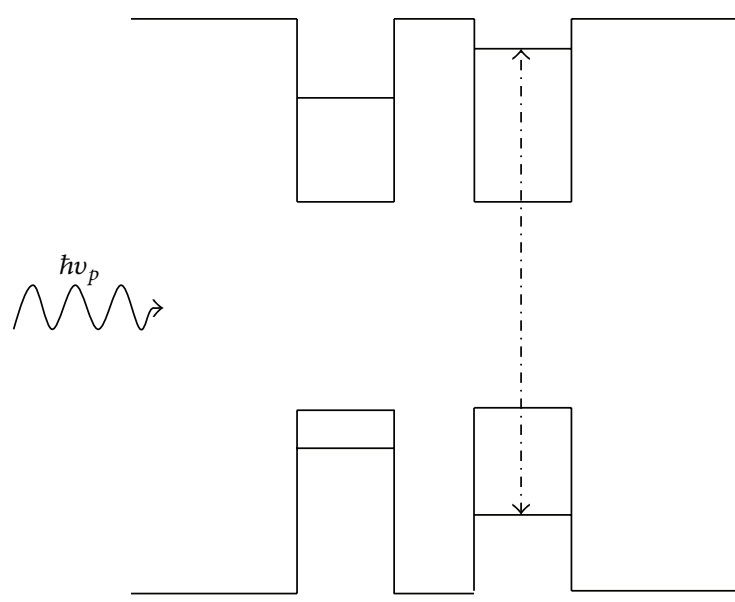

(a)

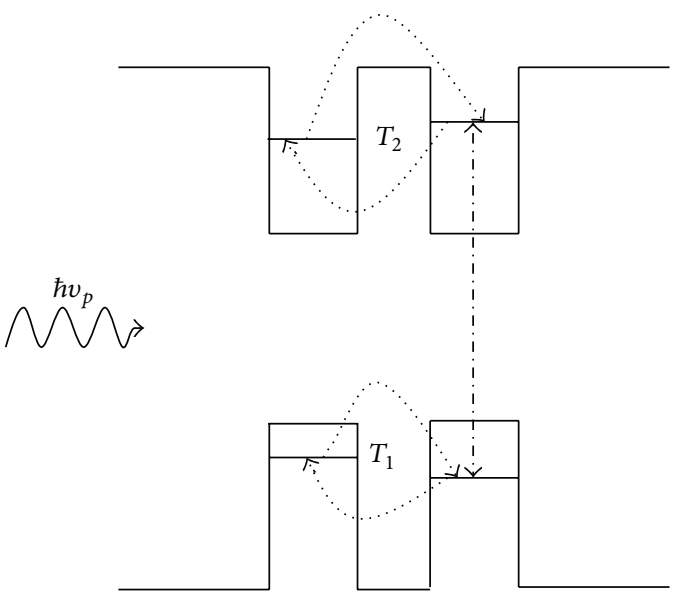

(b)

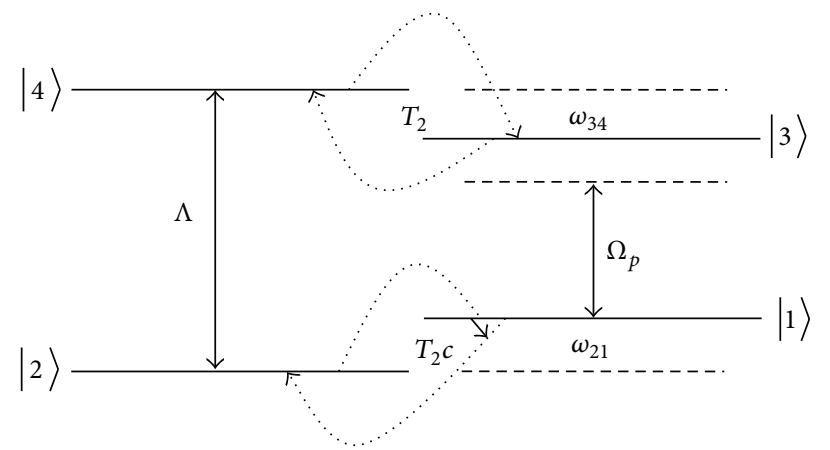

(c)

FIGURE 1: Schematic band structure and level configuration of a double QD system. (a) Without a gate voltage, electron tunneling is weak. (b) With applied gate voltage, conduction and valence band levels get into resonance, increasing their coupling. (c) Levels taken into account by Hamiltonian model and the basis of states used to describe it.

such as large electric dipole moments due to the small effective electron mass, and a great flexibility in the device design by a proper selection of the materials and their sizes. Several studies for quantum coherence and interference in QDs have been done. For instance, Chang-Hasnain et al. proposed a semiconductor optical buffer using EIT in a QD medium and theoretically predicted its performance in slow light [23]. Coherent control of the electron tunneling in an asymmetric double QD system was investigated by Villas-Boas et al. [24]. QDs and atomic vapors have similar properties, but quantum dots have more capability to provide high nonlinear optical coefficient and large electric-dipole moments. One advantage of QDs is that they provide different energy scales and physical features which can be easily varied over a wide range of values. Quantum coherence in a QD structure can be induced by applying the laser field or by electron tunneling. By applying an optical pump pulse, they have excited an electron in one of the dots, which can in turn tunnel to the second one, as controlled by external voltage [25]. In this letter, we intend to study the transmission coefficient properties as well as group velocity control of a four-level QD structure. We find that tunnel coupling has a major influence on enhancing the transmission coefficient of the probe beam as well as switching between subluminal and superluminal light propagation. The effect of incoherent pump field on transmission properties of probe field passing through the QD molecule is then discussed. The coherence induced in this structure is due to the coupling of two exciton states arising from tunnelling instead of optical pumping process [25].

\section{Model and Equations}

Figure 1(a) denotes a QD molecule that consists of a two-QD (the left and right QDs) system coupled by tunneling. Such a QD molecule can be fabricated using the self-assembled dot growth technology [24]. The asymmetric QD molecules have been detected in the double layer of InAs/GaAs structures as a realistic example [26]. This QD molecule can be assumed as a four-level atomic system as depicted in Figure 1(b). The lower valance and upper conducting band levels of the left QD are $|2\rangle$ and $|4\rangle$, respectively, while levels $|1\rangle$ and $|3\rangle$ are the lower valance and upper conducting band levels of the second QD. Inasmuch as the energy difference of two excited levels and the lower level is large, their tunneling couplings can be ignored. By applying gate voltages level $|4\rangle$ gets closer to level $|3\rangle$ and level $|1\rangle$ gets closer to level $|2\rangle$. The system configuration after applying the gate voltages comes to the form shown in Figure 1(c). In this case, with external voltages applied to gate electrodes, electrons tunneling can occur and 
a quantum dot system is acquired. A weak tunable probe field with frequency $\omega_{p}$ and Rabi-frequency $\Omega_{p}=\vec{E}_{p} \cdot \vec{\wp}_{13} / 2 \hbar$ is applied to the transition $|3\rangle \rightarrow|1\rangle$. Here $\vec{\wp}_{13}$ is the electric dipole moment and $\vec{E}_{p}$ is the amplitude of the probe field. An incoherent pump field with the pumping rate $\Lambda$ pumps the system from the lower level $|2\rangle$ to the upper level $|4\rangle$. The probe field can excite one electron from valance to conducting band in the left QD that can tunnel to the right one. Neglecting the hole tunneling, the Hamiltonian for the system can be

$$
\begin{gathered}
H=H_{0}+H_{\text {int }}, \\
H_{\text {int }}=-\frac{\hbar}{2}\left(\Omega_{p} \exp \left(i v_{p} t\right)|1\rangle\left\langle 3\left|+T_{1}\right| 1\right\rangle\langle 2|\right. \\
\left.+T_{2}|3\rangle\langle 4|\right)+ \text { c.c. }
\end{gathered}
$$

The density-matrix approach for obtaining the dynamics of the whole system is used:

$$
\dot{\rho}=-\frac{i}{\hbar}[H, \rho]
$$

where $H$ is the Hamiltonian of the QD system. Substituting (1) into (2) and after moving to an appropriate rotating frame,

$$
\begin{gathered}
\rho_{31}=\tilde{\rho}_{31} e^{-i v_{p} t}, \quad \rho_{i j}=\tilde{\rho}_{i j} \quad(i \neq 3, j \neq 1), \\
\rho_{i i}=\tilde{\rho}_{j j} \quad(i, j=1,2,3,4)
\end{gathered}
$$

we can obtain the set of density matrix equations of motion:

$$
\begin{aligned}
& \dot{\tilde{\rho}}_{13}=-\left(\frac{\Gamma_{31}}{2}-i \Delta_{p}\right) \tilde{\rho}_{13}-i \Omega_{p}\left(\tilde{\rho}_{33}-\tilde{\rho}_{11}\right) \\
& -i\left(T_{1} \tilde{\rho}_{23}-T_{2} \widetilde{\rho}_{14}\right) \text {, } \\
& \dot{\tilde{\rho}}_{14}=-\left[\frac{1}{2}\left(\Gamma_{41}+\Lambda\right)-i\left(\Delta_{p}-\omega_{34}\right)\right] \widetilde{\rho}_{14}-i \Omega_{p} \widetilde{\rho}_{34} \\
& -i\left(T_{1} \tilde{\rho}_{24}-T_{2} \widetilde{\rho}_{13}\right) \text {, } \\
& \dot{\tilde{\rho}}_{24}=-\left[\frac{1}{2}\left(\Gamma_{42}+2 \Lambda\right)-i\left(\Delta_{p}-\left(\omega_{21}+\omega_{34}\right)\right)\right] \tilde{\rho}_{24} \\
& -i\left(T_{1} \tilde{\rho}_{14}-T_{2} \tilde{\rho}_{23}\right) \text {, } \\
& \dot{\tilde{\rho}}_{34}=-\left[\frac{1}{2}\left(\Gamma_{43}+\Lambda\right)+i \omega_{34}\right] \tilde{\rho}_{34}-i \Omega_{p} \tilde{\rho}_{14} \\
& -i T_{2}\left(\tilde{\rho}_{44}-\tilde{\rho}_{33}\right) \text {, } \\
& \dot{\tilde{\rho}}_{23}=-\left[\frac{1}{2}\left(\Gamma_{32}+\Lambda\right)-i\left(\Delta_{p}-\omega_{21}\right)\right] \tilde{\rho}_{23}+i \Omega_{p} \tilde{\rho}_{21} \\
& -i\left(T_{1} \widetilde{\rho}_{13}-T_{2} \widetilde{\rho}_{24}\right) \text {, } \\
& \dot{\tilde{\rho}}_{12}=-\left[\frac{1}{2}\left(\Gamma_{12}+\Lambda\right)-i \omega_{21}\right] \widetilde{\rho}_{12}-i \Omega_{p} \widetilde{\rho}_{32}-i T_{1}\left(\widetilde{\rho}_{22}-\widetilde{\rho}_{11}\right) \text {, } \\
& \dot{\tilde{\rho}}_{22}=-\Lambda \tilde{\rho}_{22}+\left(\gamma_{42}+\Lambda\right) \tilde{\rho}_{44}+\gamma_{32} \widetilde{\rho}_{33}+\gamma_{12} \widetilde{\rho}_{11} \\
& -i T_{1}\left(\tilde{\rho}_{12}-\tilde{\rho}_{21}\right) \text {, }
\end{aligned}
$$

$$
\begin{aligned}
& \dot{\tilde{\rho}}_{33}=-\left(\gamma_{31}+\gamma_{32}\right) \tilde{\rho}_{33}-i \Omega_{p}\left(\tilde{\rho}_{13}-\tilde{\rho}_{31}\right)-i T_{2}\left(\tilde{\rho}_{43}-\tilde{\rho}_{34}\right) \\
& \dot{\tilde{\rho}}_{44}=\Lambda \tilde{\rho}_{22}-\left(\gamma_{41}+\gamma_{42}+\Lambda\right) \tilde{\rho}_{44}-i T_{2}\left(\tilde{\rho}_{34}-\tilde{\rho}_{43}\right) \\
& \tilde{\rho}_{11}+\widetilde{\rho}_{22}+\tilde{\rho}_{33}+\tilde{\rho}_{44}=1
\end{aligned}
$$

where $\Delta_{p}=v_{p}-\omega_{13}$ is the frequency detuning of the probe field, while $v_{p}$ is the frequency of the weak probe field.

The life time broadening $\gamma_{i}$ and dephasing broadening line widths $\Gamma_{i}$ are added phenomenologically in the above density matrix equations. Usually, $\Gamma_{i}$ is the dominant mechanism. Originally, (3a)-(3b) for the density matrix $\rho$ are

$$
\frac{\partial \rho}{\partial t}=-\frac{i}{\hbar}[H, \rho]+\left.\frac{\partial \rho}{\partial t}\right|_{e-e} ^{\text {incoh }}+\left.\frac{\partial \rho}{\partial t}\right|_{e-p} ^{\text {incoh9 }},
$$

where $H$ is the Hamiltonian of the QD system. The second and third terms are the incoherent contributions of the electron-electron and electron-phonon scattering processes to the system's dynamics. These terms are responsible for the damping in the system. The electron density is assumed to be high here; therefore, the dynamic screening will be strong. As a result, we only consider the dephasing terms caused by these processes. We have also ignored the electronelectron scattering between electrons in different conduction subbands, because the energy spacings between the subbands are considered to be large. Also, the damping rates caused by the electron-electron scattering processes in the upper subband are ignored, because (1) the upper subbands are separated from each other and the ground subband by energies greater than that of LO phonons and therefore excited electrons are affected by the very fast electron LOphonon scattering process, and (2) the electron envelope functions in the upper subbands are asymmetric and very much less localized in the well than those in the ground subband. Generally, in SQWs the overall decay rates are given by $\gamma_{i}=\gamma_{i j}+\gamma_{i j}^{\mathrm{dph}}$. The former $\gamma_{i j}$ denotes the lifetime broadening linewidth, which is due primarily to longitudinal optical photon emission at low temperature. The latter $\gamma_{i j}^{\mathrm{dph}}$ is the dephasing broadening linewidth, which may originate from electron-electron scattering and electronphonon scattering, as well as inhomogeneous broadening due to scattering on interface roughness. Generally, $\gamma_{i j}^{\mathrm{dph}}$ is the dominant mechanism in a semiconductor solid state system in contrast to the atomic medium.

The terms contain $T_{1}$ and $T_{2}$ in density matrix equations which show the effect of interd ot tunneling between two QDs. Through a semiclassical analysis, the set of (3a)-(3b) can be solved numerically to obtain the steady state response of the medium. In fact, response of the medium is determined by the susceptibility $\chi_{p}$ of the probe field for the $|3\rangle \rightarrow|1\rangle$ transition, which is defined as

$$
\chi\left(\omega_{p}\right)=\frac{N\left|\wp_{13}\right|^{2} \rho_{31}}{2 \hbar \varepsilon_{0} \Omega_{p}},
$$

where $N$ is the atomic density number in the medium. It is worth pointing out that the gain or absorption coefficient 


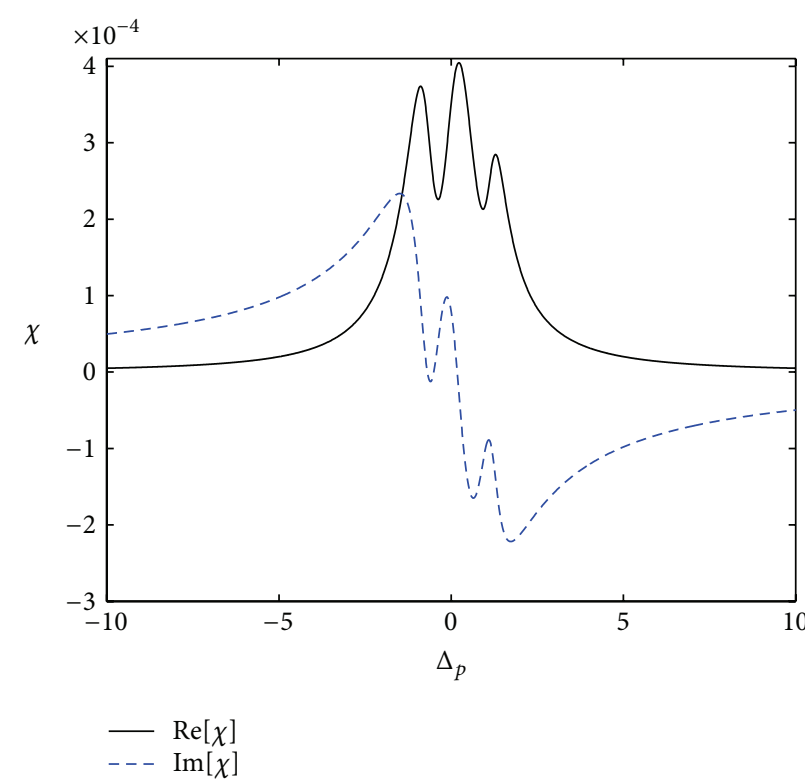

(a)

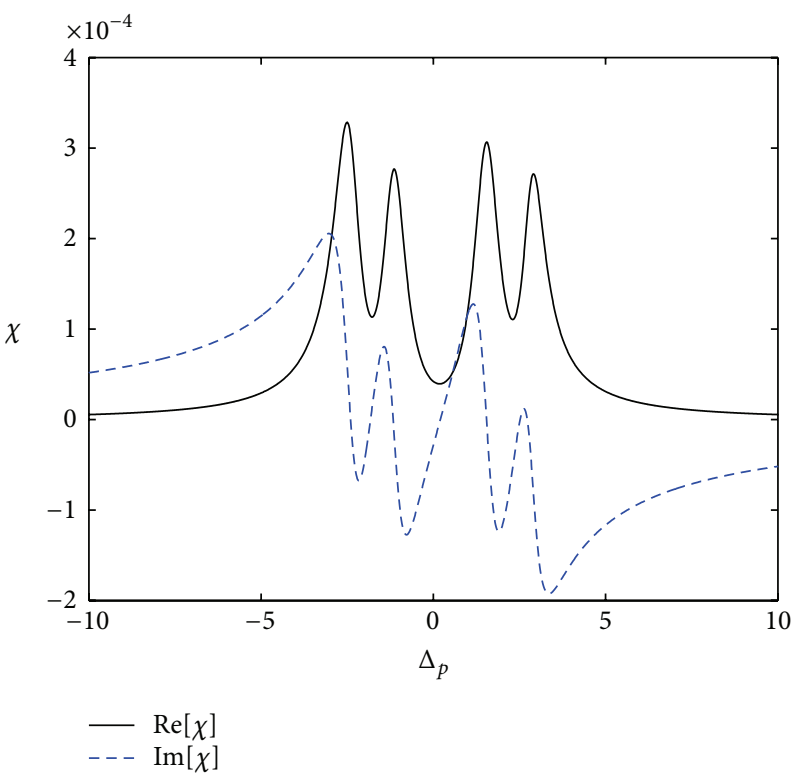

(c)

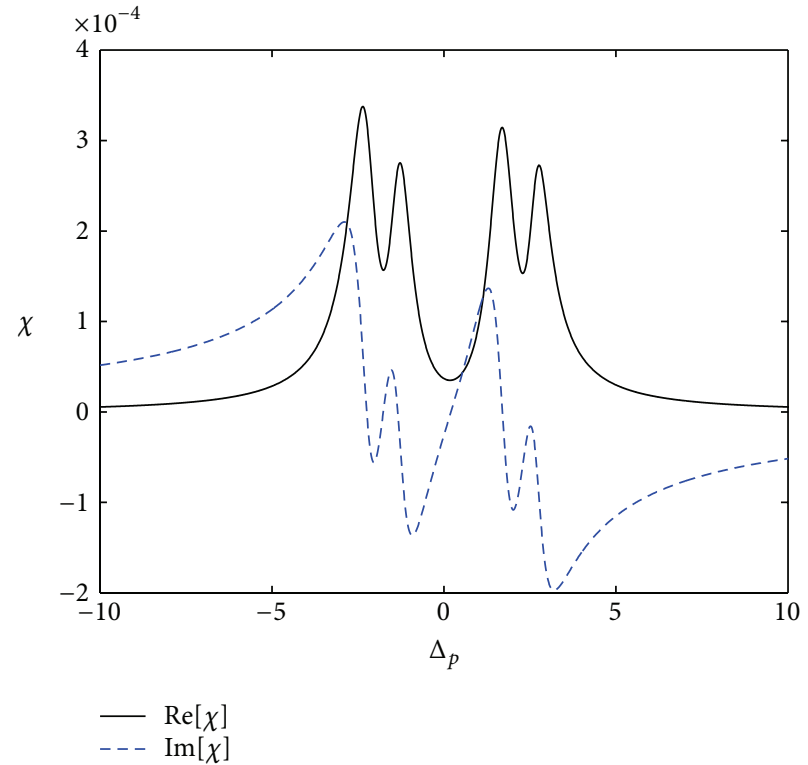

(b)

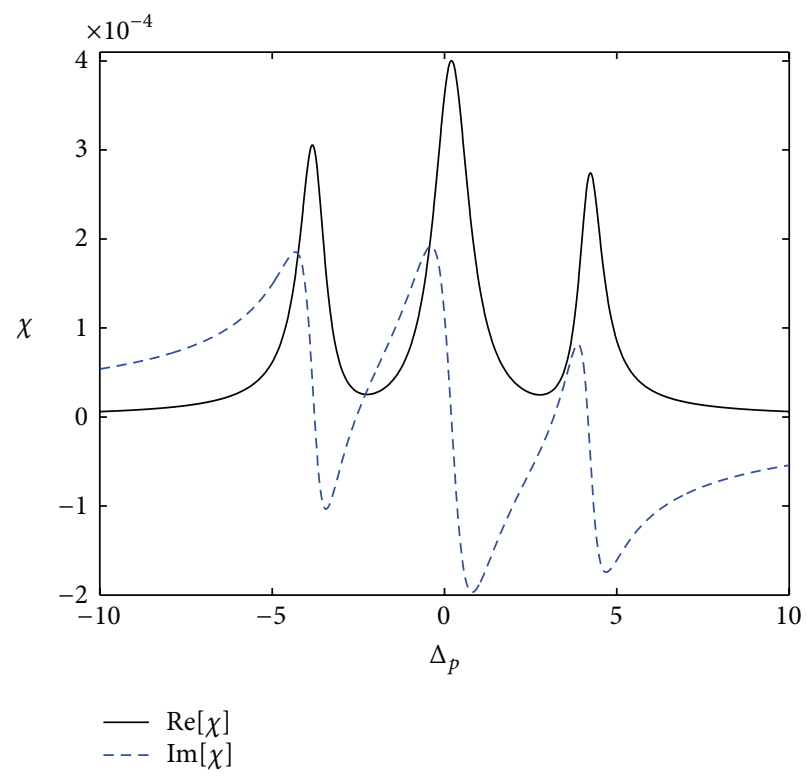

(d)

FIGURE 2: Real and imaginary parts of the linear susceptibility versus probe field detuning. The selected parameters are (a) $T_{1}=T_{2}=0.5 \mathrm{meV}$; (b) $T_{1}=2 \mathrm{meV}, T_{2}=0.5 \mathrm{meV}$; (c) $T_{1}=0.5 \mathrm{meV}, T_{2}=2 \mathrm{meV}$; (d) $T_{1}=T_{2}=2 \mathrm{meV}$. The other parameters are $\gamma_{41}=\gamma_{42}=\gamma_{31}=\gamma_{32}=0.1 \mathrm{meV}$, $\Gamma_{12}=5 \mathrm{meV}, \Gamma_{31}=2 \mathrm{meV}, \Gamma_{41}=\Gamma_{42}=\Gamma_{32}=0.5 \mathrm{meV}, \Gamma_{43}=2 \mathrm{meV}, \gamma_{12}=0.05 \mathrm{meV}, \omega_{21}=\omega_{34}=0.2 \mathrm{meV}$, and $\Lambda=0, \Omega_{p}=0.001 \mathrm{meV}$.

for the probe field is proportional to the imaginary part of susceptibility, while the real part of susceptibility corresponds to the dispersion $[8,11,12,19]$.

\section{Results and Discussion}

We now present details of the interdot tunneling on optical absorption-dispersion, group index, and transmission coefficient spectra of the medium based on (3b), (4b).

3.1. Absorption-Dispersion of the Weak Probe Field. For this four-level QD medium, we plot the real and imaginary parts of susceptibility $\chi$ versus $\Delta_{p}$ for different values of $T_{1}$ and $T_{2}$ in Figure 2. From Figure 2(a) it is found that when $T_{1}=$ $T_{2}=0.5 \mathrm{meV}$, there are three peaks in the absorption spectra. The slope of dispersion around $\Delta_{p}=0$ is negative that is accompanied with a large absorption, while the slope of dispersion on both sides of zero probe detuning is positive. Figure 2(b) shows that for $T_{1}=2 \mathrm{meV}, T_{2}=0.5 \mathrm{meV}$, the number of absorption peaks becomes four. In this case, the absorption will be decreased at line center of probe detuning and a transparency window appears. In addition, the slope of dispersion converts to positive at $\Delta_{p}=0$. The effect of electron tunneling $T_{2}$ on linear susceptibility is depicted 


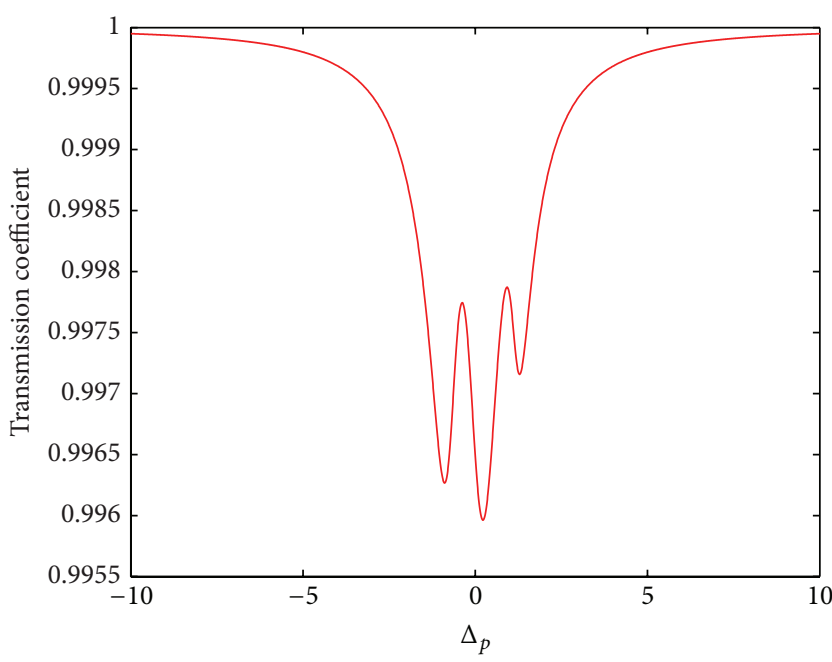

(a)

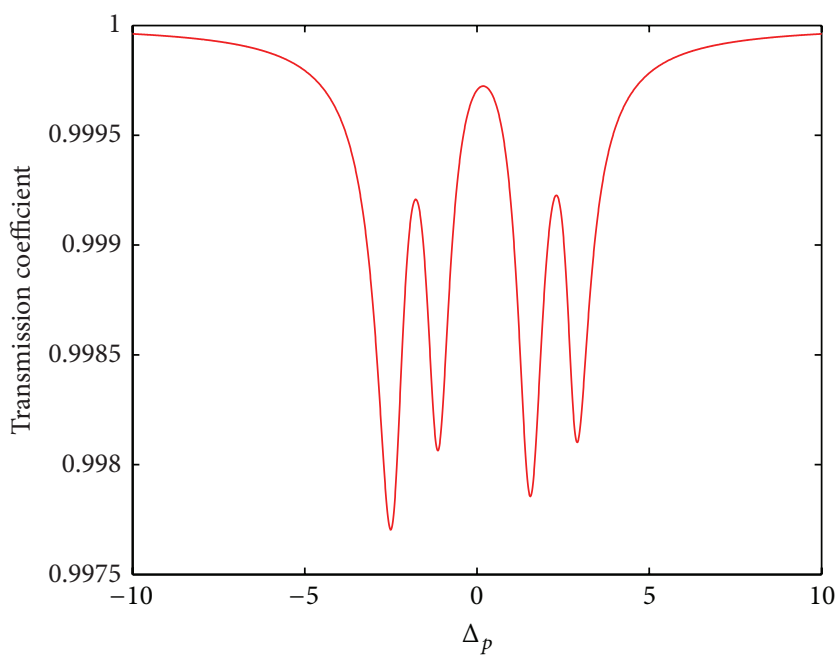

(c)

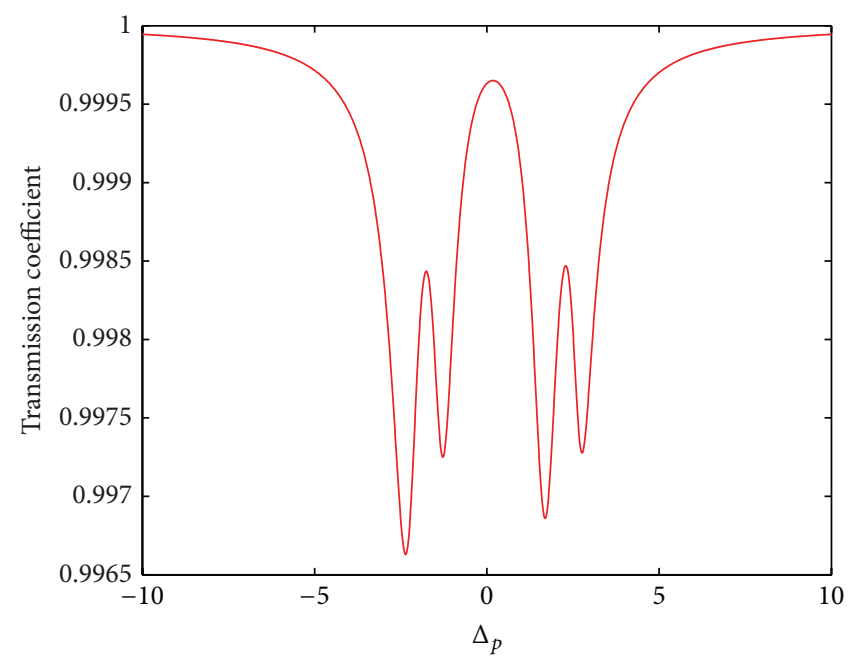

(b)

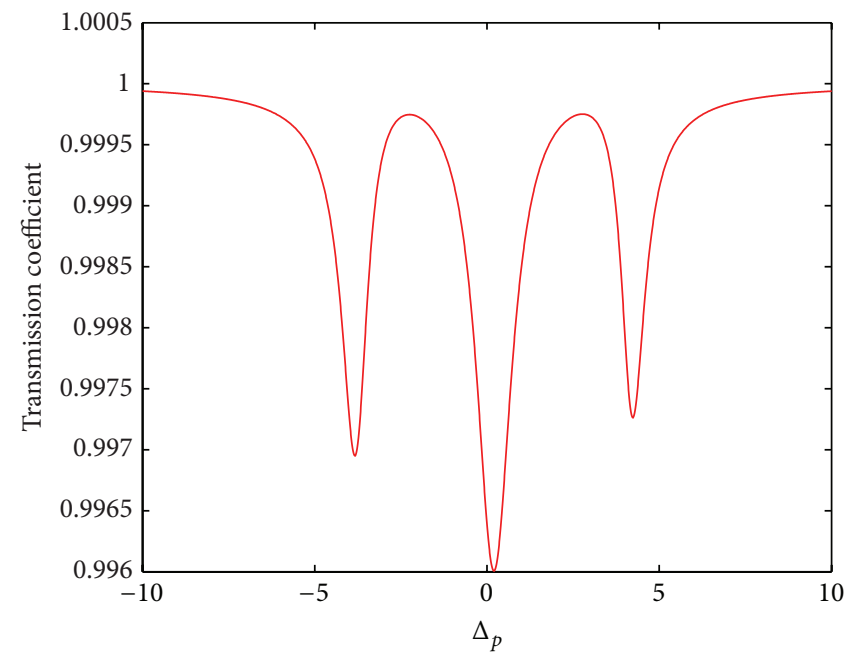

(d)

Figure 3: Transmission coefficient versus probe field detuning. The selected parameters are (a) $T_{1}=T_{2}=0.5 \mathrm{meV}$; (b) $T_{1}=2 \mathrm{meV}, T_{2}=$ $0.5 \mathrm{meV}$; (c) $T_{1}=0.5 \mathrm{meV}, T_{2}=2 \mathrm{meV}$; (d) $T_{1}=T_{2}=2 \mathrm{meV}$. The other parameters are the same as in Figure 2.

in Figure 2(c). It is clear that absorption and dispersion of the probe field in Figure 2(c) is similar to Figure 2(b). Thus, it is deduced that when electron tunneling parameters $T_{1}$ and $T_{2}$ impact separately on real and imaginary parts of susceptibility, uniform results are obtained. The curves of absorption and dispersion spectra for $T_{1}=T_{2}=2 \mathrm{meV}$ are plotted in Figure 2(d). It is obvious that three-peak spectral feature appears again and the value of probe absorption at $\Delta_{p}=0$ becomes very large which separates two transparency windows. Simultaneously, the slope of dispersion at line center of probe detuning switches again to negative which is corresponding to superluminal light propagation.

3.2. Transmission Coefficient of the Weak Probe Field. Now, we display the transmission properties of the probe field propagating through the QD medium in Figure 3 for different values of electron tunneling parameters. An investigation in Figure 3(a) shows that for the case of $T_{1}=T_{2}=0.5 \mathrm{meV}$, a low transmission appears at $\Delta_{p}=0$, accompanied with two sideband dips (Figure 3(a)). In the first step, we discuss the effect of each term of electron tunneling, that is, $T_{1}$ and $T_{2}$, on optical transmission coefficient spectra of the QD medium separately. The results for two different cases of $T_{1}=2 \mathrm{meV}$, $T_{2}=0.5 \mathrm{meV}$ and $T_{1}=0.5 \mathrm{meV}, T_{2}=2 \mathrm{meV}$ are displayed in Figures 3(b) and 3(c). It can be seen from the figures that the number of transmission dips is split to four. Moreover, a high transmission coefficient takes place at $\Delta_{p}=0$. In order to further illustrate explicitly the combined influence of both electron tunneling parameters on transmission properties of the probe beam, we plot the transmission coefficient spectra of the probe field as a function of probe field detuning in Figure 3(d). It is shown that the number of transmission spectral dips is again three. A low transmission appears at $\Delta_{p}=0$ which corresponds to a high absorption. Results are in a good agreement with our presented results in Section 3.1 for absorption properties of the probe field in the QD medium. 


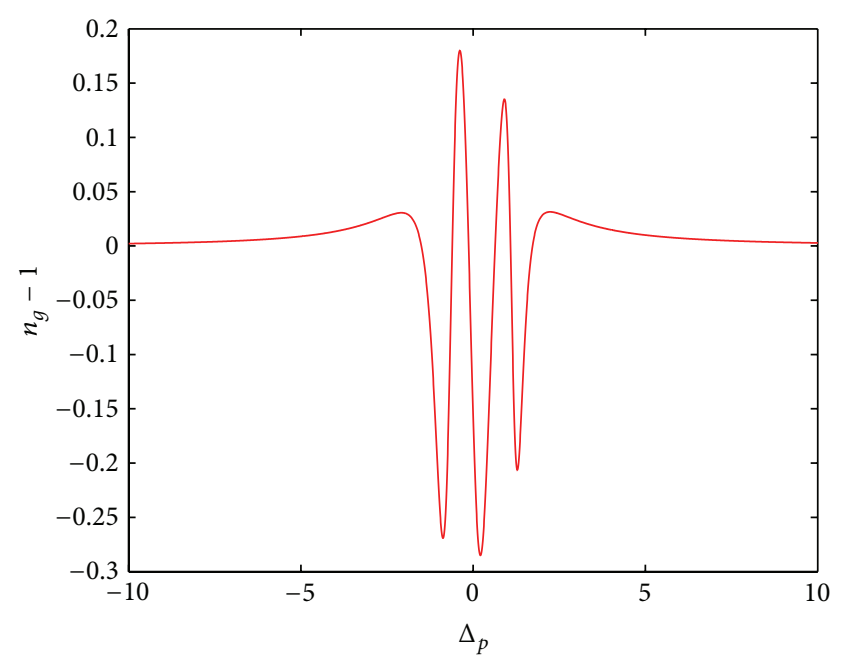

(a)

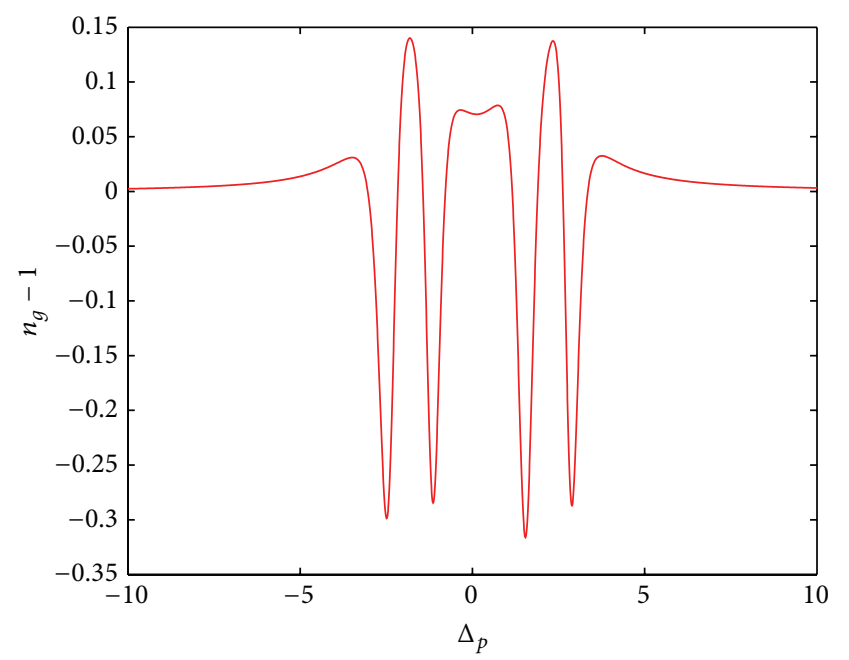

(c)

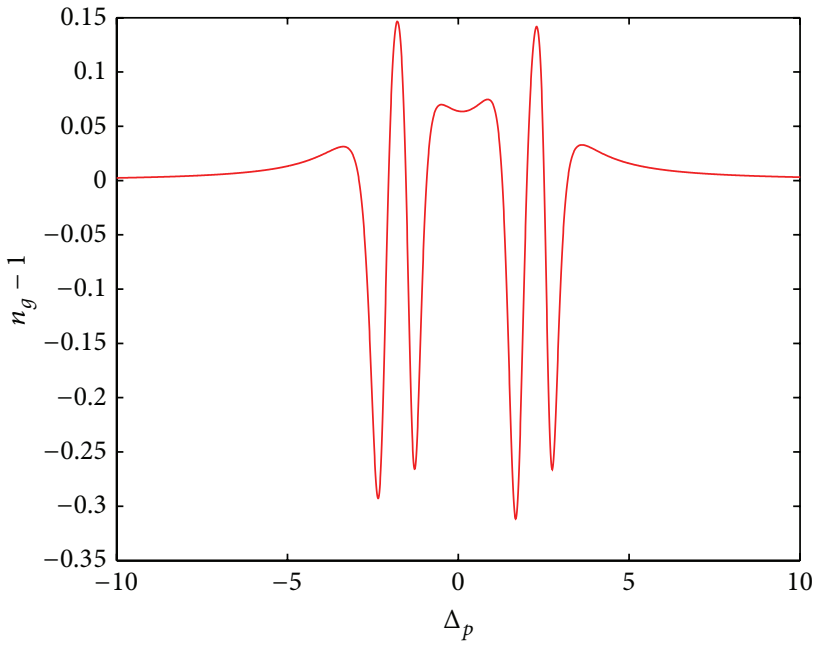

(b)

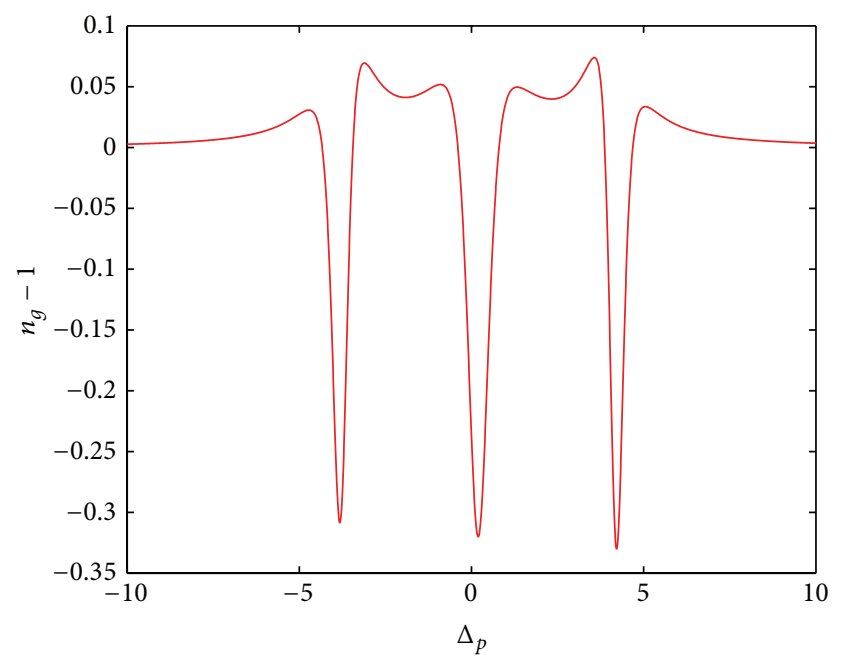

(d)

Figure 4: Group index versus probe field detuning. The selected parameters are (a) $T_{1}=T_{2}=0.5 \mathrm{meV}$; (b) $T_{1}=2 \mathrm{meV}, T_{2}=0.5 \mathrm{meV}$; (c) $T_{1}=0.5 \mathrm{meV}, T_{2}=2 \mathrm{meV}$; (d) $T_{1}=T_{2}=2 \mathrm{meV}$. The other parameters are the same as in Figure 2.

3.3. Group Velocity of the Weak Probe Field. The slope of dispersion with respect to the probe field detuning has a major role in the group velocity of probe field. For further discussion one has to consider the relevant quantity of group index $n_{g}=c / v_{g}$, where $c$ is the speed of light in the vacuum and the group velocity $v_{g}$ is given by [27-30]

$$
v_{g}=\frac{c}{1+2 \pi \operatorname{Re}\left(\chi\left(v_{p}\right)\right)+2 \pi v_{p}\left(\partial / \partial v_{p}\right) \operatorname{Re}\left(\chi\left(v_{p}\right)\right)} .
$$

It is well known that the group velocity of the probe field strongly depends on the slope of dispersion. Note that a positive peak in the curves corresponds to subluminal pulse propagation, while a negative dip corresponds to superluminal pulse propagation. The group index behavior of weak probe field is shown in Figure 4. It is found from Figure 4(a) that for $T_{1}=T_{2}=0.5 \mathrm{meV}$, the group velocity corresponds to superluminal light propagation at $\Delta_{p}=0$. The effect of each term of electron tunnelings, while the other is keeping constant on group index is plotted in Figures 4(b) and 4(c) separately. It is found that the group index in these cases represents subluminal light propagation at the line center of probe field detuning. Thus, the superluminal light propagation can be switched to subluminal light propagation just by increasing $T_{1}$ or $T_{2}$ in the absence of probe absorption. At the end, the group index curve for the case $T_{1}=T_{2}=$ $2 \mathrm{meV}$ is plotted in Figure 4(d). We find that the subluminal light propagation can be converted to superluminal light propagation at zero probe field detuning. The obtained results confirm our previous curves for absorption spectra of the medium.

3.4. Effect of Incoherent Pump Field on Probe Transmission of the Weak Probe Field. Now, we will analyze how the rate of incoherent pumping affects the probe transmission of the QD medium. We find that the probe transmission exhibits oppositional features for weak and strong incoherent pump 


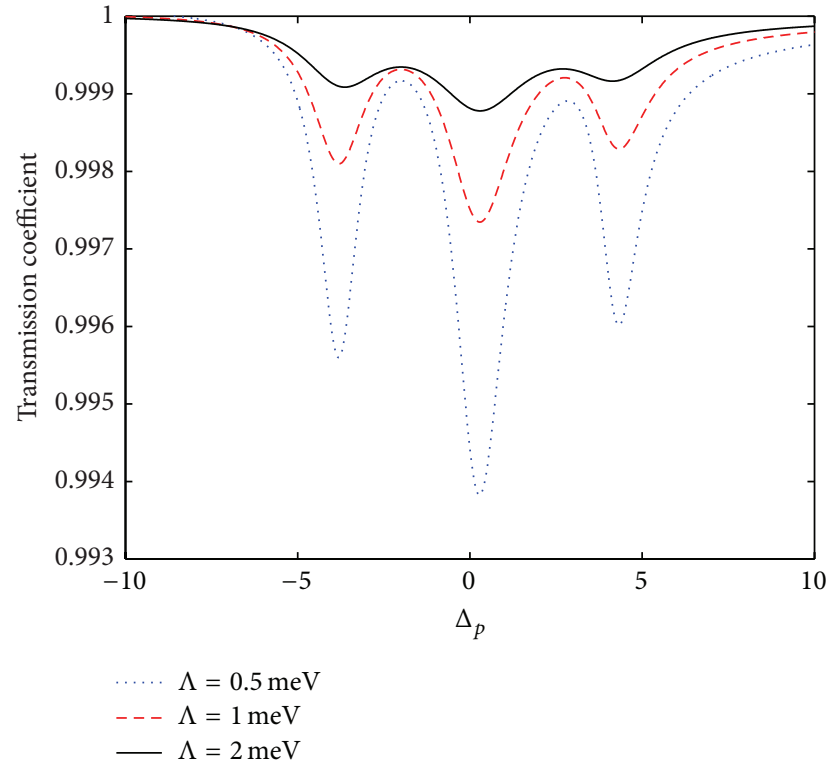

(a)

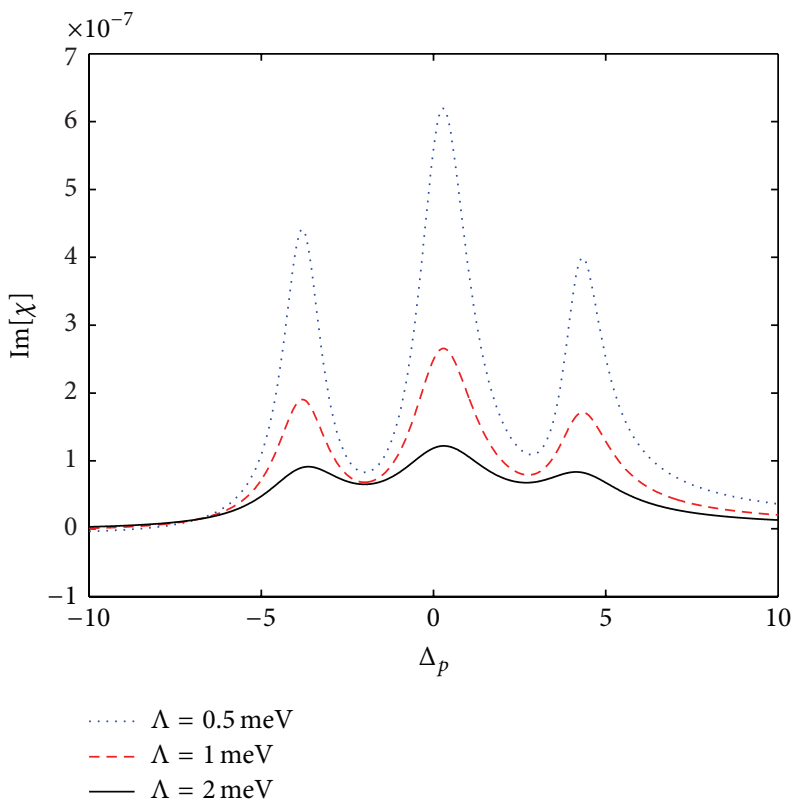

(c)

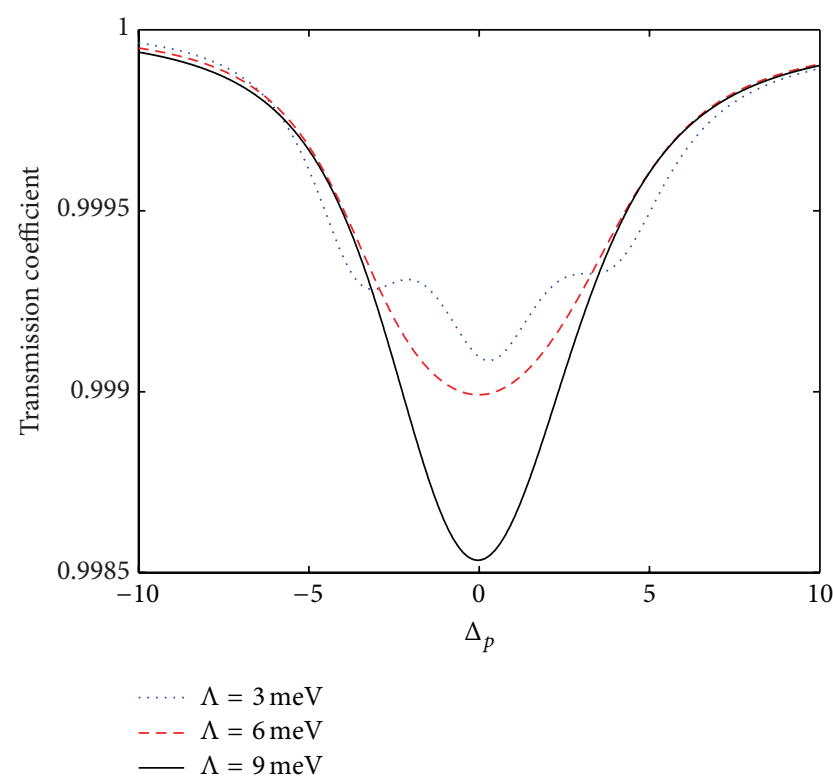

(b)

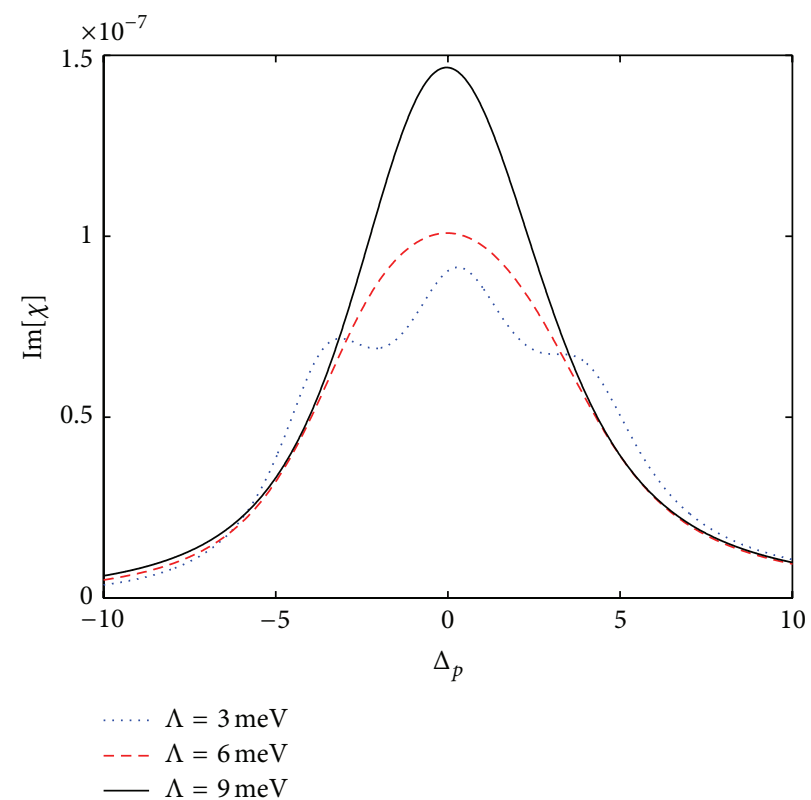

(d)

Figure 5: Transmission coefficient ((a), (b)) and probe absorption ((c), (d)) versus probe field detuning. for different values of incoherent pumping. The selected parameters are $T_{1}=T_{2}=2 \mathrm{meV}$ and the other parameters are the same as in Figure 2.

rates. We demonstrate the dependence of probe transmission on the weak (Figure 5(a)) and strong (Figure 5(b)) incoherent pump field. Clearly, for the small rate of incoherent pumping, the probe transmission enhances by increasing $\Lambda$ (Figure 5(a)) and reaches nearly 0.999. Transmission coefficient of probe field versus $\Delta_{p}=0$ and for strong incoherent pump rates is plotted in Figure 5(b). We find reduction of probe transmission so long as $\Lambda$ increases, so that the value of transmission coefficient for $\Lambda=9 \mathrm{meV}$ approximates to 0.9985 . For a physical description of such behavior, we should refer to the absorption spectra of the probe field
(Figures 5(c) and 5(d)). Physically, in the realm of weak incoherent pump fields, increasing an incoherent pump rate pumps more population from lower level $|2\rangle$ to upper level $|4\rangle$ leading to reduction of probe field absorption and thus results in enhancement of probe transmission. In other words, when the incoherent pump becomes stronger, more population will be pumped to level $|4\rangle$, which means less chances of absorption of the probe field. This condition will be different for the case of strong incoherent pump rates. Hence, although incoherent pump field brings the population from lower level $|2\rangle$ to upper level $|4\rangle$, coherence between two levels $|2\rangle$ and 
$|4\rangle$ will be destroyed by the strong incoherent pumping field. Consequently, population in level $|4\rangle$ cannot be trapped and will be accumulated in lower levels. More population in lower levels means more chances of absorption of the probe field which can lead to reduction of probe transmission $[19,21]$.

\section{Conclusion}

To summarize, in this letter, we investigated the controllable transmission coefficient and group index of a four-level QD system. The effect of electron coupling as well as incoherent pumping in light propagation is then discussed. It is found that the group velocity of light pulse can be controlled with the electron tunneling. We show that by increasing the electron tunneling parameter the superluminal light propagation can be switched to subluminal light propagation or vice versa. It is worth pointing out that no external laser field is used at the pumping processes of the system.

\section{Conflict of Interests}

The authors declare that there is no conflict of interests regarding the publication of this paper.

\section{Acknowledgment}

The presented work has been supported by the Lithuanian Research Council (No. VP1-3.1-ŠMM-01-V-03-001).

\section{References}

[1] E. Arimondo and G. Orriols, "Nonabsorbing atomic coherences by coherent two-photon transitions in a three-level optical pumping," Lettere Al Nuovo Cimento, vol. 17, no. 10, pp. 333-338, 1976.

[2] G. Alzetta, A. Gozzini, L. Moi, and G. Orriols, "An experimental method for the observation of r.f. transitions and laser beat resonances in oriented Na vapour," Il Nuovo Cimento B, vol. 36, no. 1, pp. 5-20, 1976.

[3] M. O. Scully and M. Fleischhauer, "Lasers without inversion," Science, vol. 263, no. 5145, pp. 337-338, 1994.

[4] S. E. Harris, "Electromagnetically induced transparency," Physics Today, vol. 50, no. 7, p. 36, 1997.

[5] Y. Wu and X. Yang, "Electromagnetically induced transparency in $-\vee,-\wedge$, and cascade-type schemes beyond steady-state analysis," Physical Review A, vol. 71, no. 5, Article ID 053806, 2005.

[6] M. Xiao, Y. Q. Li, S. Z. Jin, and J. Gea-Banacloche, "Measurement of dispersive properties of electromagnetically induced transparency in rubidium atoms," Physical Review Letters, vol. 74, no. 5, pp. 666-669, 1995.

[7] S. E. Harris, "Lasers without inversion: interference of lifetimebroadened resonances," Physical Review Letters, vol. 62, no. 9, pp. 1033-1036, 1989.

[8] H. R. Hamedi, G. Juzeliūnas, A. Raheli, and M. Sahrai, "High refractive index and lasing without inversion in an open fourlevel atomic system," Optics Communications, vol. 311, pp. 261265, 2013.

[9] Y. F. Zhu, A. I. Rubiera, and M. Xiao, "Inversionless lasing and photon statistics in a V-type atomic system," Physical Review A, vol. 53, no. 2, pp. 1065-1071, 1996.
[10] D. F. Phillips, A. Fleischhauer, A. Mair, R. L. Walsworth, and M. D. Lukin, "Storage of light in atomic vapor," Physical Review Letters, vol. 86, no. 5, pp. 783-786, 2001.

[11] H. Tajalli and M. Sahrai, "Switching from normal to anomalous dispersion via coherent field," Journal of Optics B, vol. 7, no. 6, pp. 168-173, 2005.

[12] H. Ghaforyan, H. R. Hamedi, A. Khaledi-Nasab et al., "Enhancement of Kerr nonlinearity at $\lambda=1550 \mathrm{~nm}$ in an $\mathrm{Er}^{3+}$. doped optical fiber," Journal of Modern Optics, vol. 60, no. 8, pp. 674-680, 2013.

[13] Y. Wu, M. G. Payne, E. W. Hagley, and L. Deng, "Efficient multiwave mixing in the ultraslow propagation regime and the role of multiphoton quantum destructive interference," Optics Letters, vol. 29, no. 19, pp. 2294-2296, 2004.

[14] L. Y. Wu and X. Yang, "Four-wave mixing in molecular magnets via electromagnetically induced transparency," Physical Review $B$, vol. 76, no. 5, Article ID 054425, 2007.

[15] W. Harshawardhan and G. S. Agarwal, "Controlling optical bistability using electromagnetic-field-induced transparency and quantum interferences," Physical Review A, vol. 53, no. 3, pp. 1812-1817, 1996.

[16] Z. Wang and M. Xu, "Control of the switch between optical multistability and bistability in three-level V-type atoms," Optics Communications, vol. 282, no. 8, pp. 1574-1578, 2009.

[17] H. R. Hamedi, S. H. Asadpour, M. Sahrai, B. Arzhang, and D. Taherkhani, "Optical bistability and multi-stability in a fourlevel atomic scheme," Optical and Quantum Electronics, vol. 45, no. 3, pp. 295-306, 2013.

[18] M. Mahmoudi, S. Worya Rabiei, L. Ebrahimi Zohravi, and M. Sahrai, "Absorption free superluminal light propagation in a three-level pump-probe system," Optics Communications, vol. 281, no. 18, pp. 4681-4686, 2008.

[19] M. Sahrai, R. Nasehi, M. Memarzadeh, H. Hamedi, and J. B. Poursamad, "Controlling the probe-absorption and -dispersion via quantum interference from incoherent pumping field in a four-level lambda-type system," European Physical Journal D, vol. 65, no. 3, pp. 571-579, 2011.

[20] S. Hossein Asadpour, M. Sahrai, R. Sadighi-Bonabi, A. Soltani, and H. Mahrami, "Enhancement of Kerr nonlinearity at long wavelength in a quantum dot nanostructure," Physica E, vol. 43, no. 10, pp. 1759-1762, 2011.

[21] R. Nasehi and M. Sahrai, "Optical bistability and multi-stability via voltage-controlled and the rate of incoherent pump in a quantum dot nanostructure," Journal of Luminescence, vol. 132, no. 9, pp. 2302-2306, 2012.

[22] M. Mahmoudi and M. Sahrai, "Absorption-free superluminal light propagation in a quantum-dot molecule," Physica E, vol. 41, no. 10, pp. 1772-1778, 2009.

[23] C. J. Chang-Hasnain, P. C. Ku, J. Kim, and S. L. Chuang, "Variable optical buffer using slow light in semiconductor nanostructures," Proceedings of the IEEE, vol. 91, no. 11, pp. 18841897, 2003.

[24] J. M. Villas-Boas, A. O. Govorov, and S. E. Ulloa, "Coherent control of tunneling in a quantum dot molecule," Physical Review B, vol. 69, no. 12, Article ID 125342, 2004.

[25] J. Li, R. Yu, J. Liu, P. Huang, and X. Yang, "Voltage-controlled optical bistability of a tunable three-level system in a quantumdot molecule," Physica E, vol. 41, no. 1, pp. 70-73, 2008.

[26] G. G. Tarasov, Z. Y. Zhuchenko, M. P. Lisitsa et al., "Optical detection of asymmetric quantum-dot molecules in doublelayer InAs/GaAs structures," Semiconductors, vol. 40, no. 1, pp. 79-83, 2006. 
[27] L. J. Wang, A. Kuzmich, and A. Dogariu, "Gain-assisted superluminal light propagation," Nature, vol. 406, no. 6793, pp. 277$279,2000$.

[28] O. Kocharovskaya, Y. Rostovtsev, and M. O. Scully, "Stopping light via hot atoms," Physical Review Letters, vol. 86, no. 4, pp. 628-631, 2001.

[29] C. Liu, Z. Dutton, C. H. Behroozi, and L. V. Hau, "Observation of coherent optical information storage in an atomic medium using halted light pulses," Nature, vol. 409, no. 6819, pp. 490493, 2001.

[30] D. F. Phillips, A. Fleischhauer, A. Mair, R. L. Walsworth, and M. D. Lukin, "Storage of light in atomic vapor," Physical Review Letters, vol. 86, no. 5, pp. 783-786, 2001. 

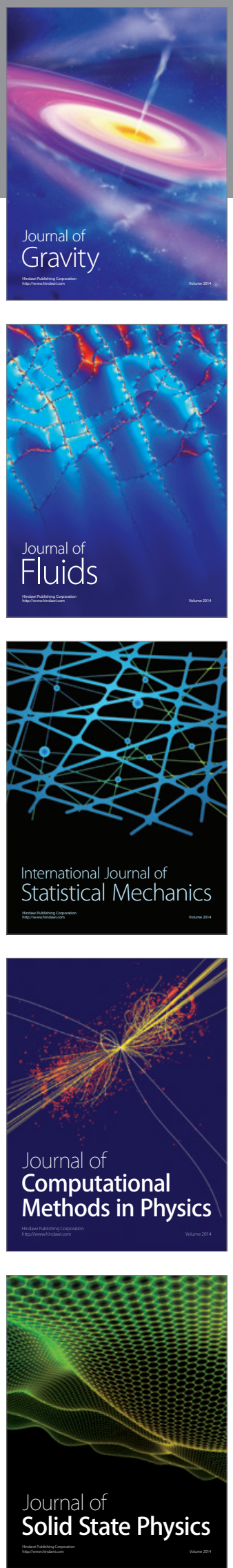

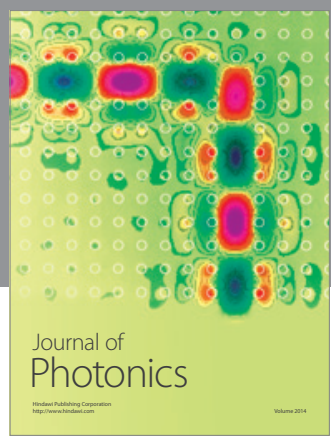

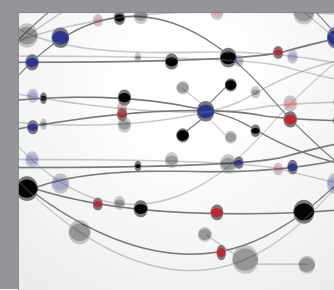

The Scientific World Journal

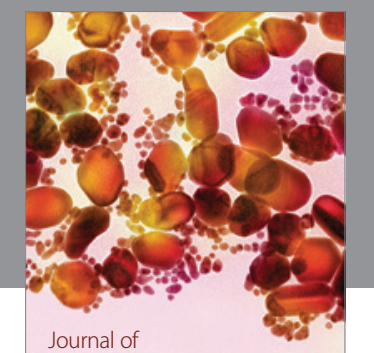

Soft Matter
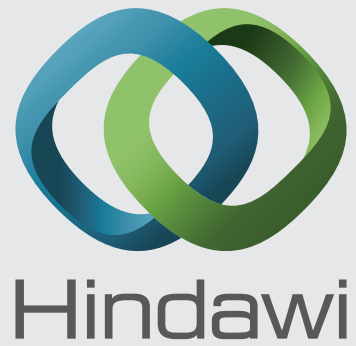

Submit your manuscripts at

http://www.hindawi.com
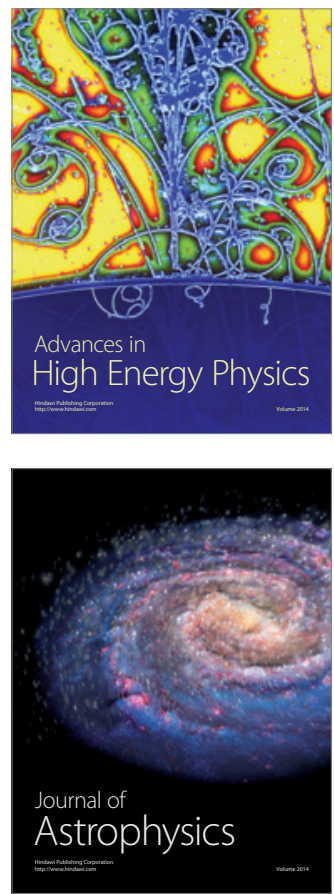
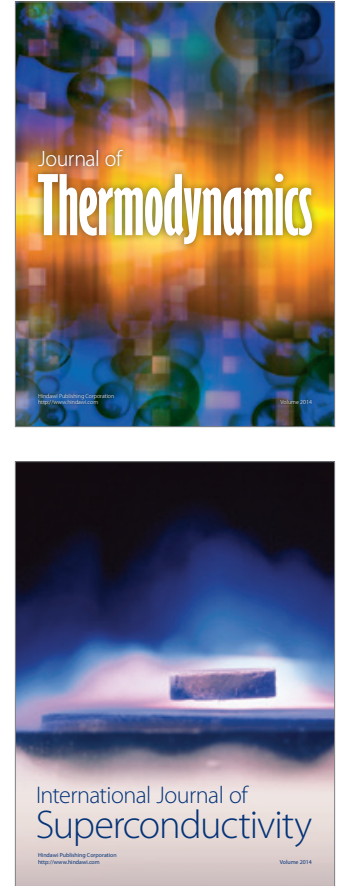
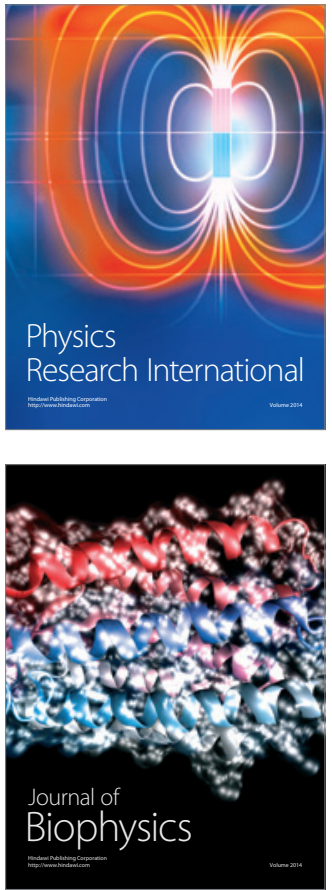
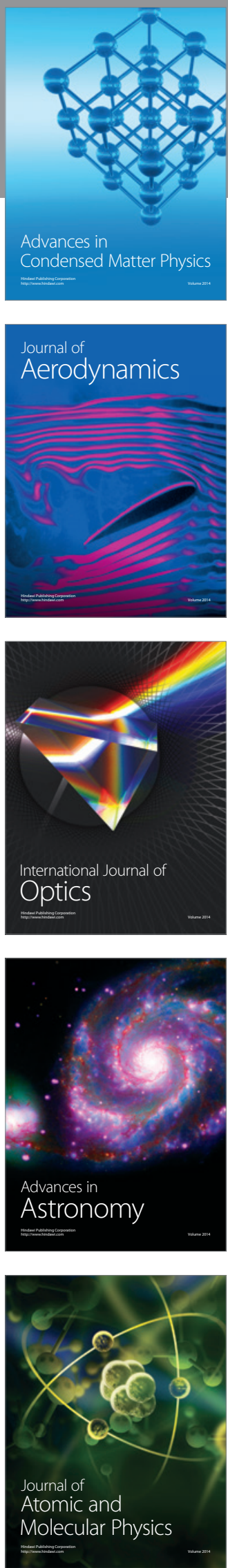